\title{
Intermediate band mobility in heavily titanium-doped silicon layers
}

\author{
G. Gonzalez-Díaz ${ }^{\mathrm{a}}$, J. Olea ${ }^{\mathrm{a}}$, I. Mártil ${ }^{\mathrm{a}}$, D. Pastor ${ }^{\mathrm{a}}$, A. Martí ${ }^{\mathrm{b}, *}$, E. Antolín ${ }^{\mathrm{b}}$, A Luque $^{\mathrm{b}}$ \\ a Dpto. de Física Aplicada III (Electricidad y Electrónica), Facultad de Ciencias Físicas, Universidad Complutense de Madrid, 28040 Madrid, Spain \\ ${ }^{\mathrm{b}}$ Instituto de Energía Solar, Universidad Politécnica de Madrid, Escuela Técnica Superior de Ingenieros de Telecomunicación, Ciudad Universitaria sn, 28040 Madrid, Spain
}

\section{A R T I C L E I N F O}

\section{Article history:}

Received 29 March 2009

Accepted 7 May 2009

Available online 29 May 2009

Keywords:

Intermediate band

Solar cells

Silicon

Titanium

Mobility

Novel concepts

\begin{abstract}
A B S T R A C T
The sheet resistance and the Hall mobility of high-purity Si wafers, in whose surface Ti atoms are implanted and laser annealed reaching concentrations above $10^{21} \mathrm{~cm}^{-3}$, are measured in the $90-370 \mathrm{~K}$ range. Below $240 \mathrm{~K}$, an unconventional behavior is observed that is well explained on the basis of the appearance of an intermediate band (IB) region able to form a blocking junction with the substrate and of the appearance of an IB conduction. Explanations based on ordinary device physics fail to justify all the unconventional behavior of the characteristics observed.
\end{abstract}

(c) 2009 Elsevier B.V. All rights reserved.

\section{Introduction}

This research has been originated within the development of the intermediate band solar cell (IBSC). The IBSC is a novel device conceived to exceed the efficiency of conventional solar cells [1,2]. The concept was first proposed in 1997 [3] and since then, efforts have been made to understand its behavior [4-10], to manufacture practical devices and to empirically demonstrate its operation principles.

The IBSC contains an intermediate band (IB) material which is characterized by the existence of an electronic energy band of allowed states within the conventional band gap $E_{G}$ (Fig. 1). This IB splits $E_{G}$ into two sub-gaps, $E_{L}$ and $E_{H}$, enabling the creation of additional electron-hole pairs by the absorption of two sub-bandgap energy photons. In this process, one photon pumps an electron from the valence band (VB) to the IB (photon 1) and a second photon (photon 2) pumps an electron from the IB to the conduction band (CB). To ensure the occurrence of both processes, the IB must be a semi-filled or "metallic" band. Since the process described occurs additionally to the ordinary pumping of electrons from the $\mathrm{VB}$ to the $\mathrm{CB}$ by photons of enough energy (photon 3), the solar cell photocurrent is higher than in the case of a conventional design.

In order to obtain high efficiencies, this increase in current has to be achieved without substantial reduction of the voltage. This implies that, besides the conventional quasi-Fermi levels $E_{F, C}$ and $E_{F, V}$ for the $\mathrm{CB}$ and $\mathrm{VB}$, a third quasi-Fermi level $E_{F, I}$ associated to

\footnotetext{
* Corresponding author. Tel.: +34914533551; fax: +34915446341.

E-mail address: amarti@etsit.upm.es (A. Martí).
}

the electron population in the IB appears when the solar cell is in operation. As it is also shown in Fig. 1, the split between quasiFermi levels, which determines the output voltage of the cell, is ensured by sandwiching the IB material in-between two emitters of conventional semiconductor, one $p$ type and the other $n$ type [4].

The efficiency limit of IBSCs designed according to the exposed model is very high, over 63\% [3], to be compared to the limit efficiency of single gap solar cells calculated by Shockley and Queisser in 1961, which is $40.7 \%$ under the same conditions [1,2]. Regarding the practical implementation of the concept, IBSC prototypes have been manufactured using quantum dot (QD) arrays embedded in a higher band-gap semiconductor matrix $[5,6,8,11,12]$. In this approach, the IB is formed by the QD-confined levels and it can be semi-filled by doping [10]. These QD-IBSCs have provided experimental proof of two key principles of the IBSC model, namely, the electron-hole generation by double subbandgap photon absorption [13] and the triple quasi-Fermi level splitting [14].

Alternatively to QDs, it has also been suggested [15] that actually any element which produces deep levels in a given semiconductor can be used, in principle, to manufacture an IB material. However, it is known long ago that deep levels are a major cause of SRH non-radiative recombination in semiconductors $[16,17]$ which is detrimental for solar cells. However, if the impurity concentration is increased above the Mott transition [15], then the SRH recombination is expected to be suppressed.

The purpose of this paper is to study some electrical properties of this IB when this is implemented from deep centers. For it we use $\mathrm{Ti}$, one of the most promising materials to obtain an IB in a variety of hosts [18-21]. In our work, the Ti is dissolved in Si at 


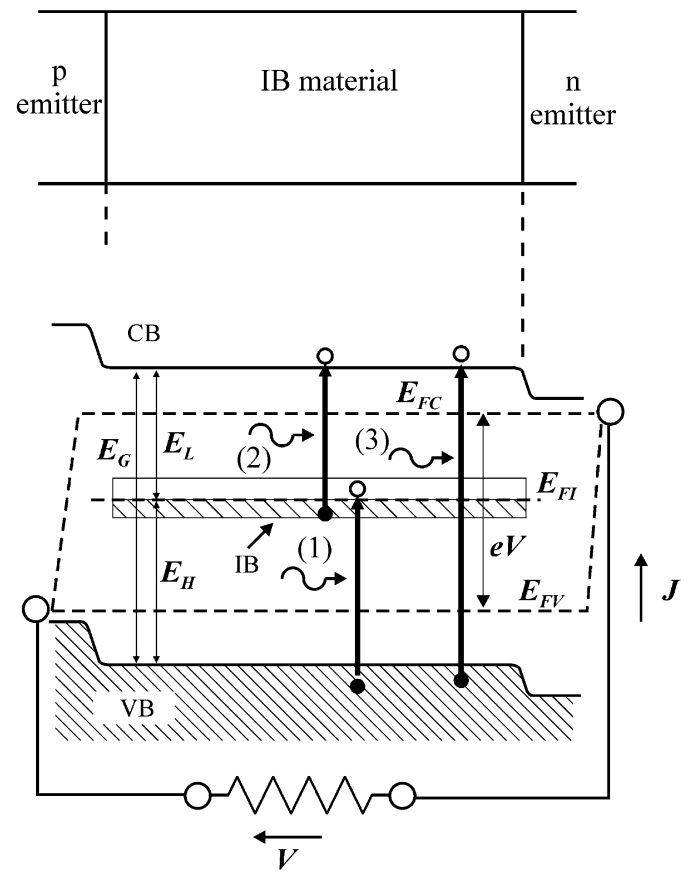

Fig. 1. Illustration of the fundamental operation of the intermediate-band solar cell.

high concentrations. It is known that at small concentrations, it introduces deep donor energy levels at a distance from the conduction band in the order of 0.3 [22-26] or even $0.51 \mathrm{eV}$ [27]. An acceptor, which lays $0.08 \mathrm{eV}$ below the $\mathrm{CB}$ and is thus fairly inactive $[25,27]$, has also been identified. But the solubility values of $\mathrm{Ti}$ in $\mathrm{Si}$ reported in the literature lie far below the range required for exceeding the Mott transition, at about $6 \times 10^{19} \mathrm{~cm}^{-3}$ at $300 \mathrm{~K}$ [15], and therefore for the formation of a true IB with suppressed SRH recombination. To overcome this drawback, the pulsed laser melting (PLM) preparation method has been used [28], which is known to succeed in incorporating shallow dopants to the Si lattice at concentrations far above the equilibrium solid solubility [29]. This method has also been successfully used for the preparation of the IB materials based on the band anticrossing mechanism [30].

\section{Sheet resistance and mobility}

Si (111) wafers $300 \mu \mathrm{m}$ thick of high resistivity $(200 \Omega \mathrm{cm}$, light $n$-doping $N_{\mathrm{D}}=2.25 \times 10^{13} \mathrm{~cm}^{-3}$ ), have been implanted with $\mathrm{Ti}$ ions [28] at ultrahigh doses $\left(10^{15}\right.$ and $\left.5 \times 10^{15} \mathrm{~cm}^{-2}\right)$. Then, to recover the crystallinity of the implanted layers and electrically activate the Ti impurities, a $\operatorname{KrF}$ excimer laser $(\lambda=248 \mathrm{~nm})$ was used with energy densities of 0.6 and $0.8 \mathrm{~J} \mathrm{~cm}^{-2}$ and pulse width of $20 \mathrm{~ns}$. Titanium profiles have been determined by ToF SIMS measurements before and after laser irradiation. For the $10^{15} \mathrm{~cm}^{-2}$ implantation irradiated with $0.8 \mathrm{~J} \mathrm{~cm}^{-2}$, loss of Ti atoms was in the range of $40 \%$.

We present in Fig. 2 the sheet resistance and the Hall mobility of the substrate wafer and of implanted wafers on this substrate as a function of the temperature. Aluminum contacts are deposited on the top of the samples following the van der Pauw configuration scheme without any subsequent thermal treatment. Therefore, for the Hall measurements, only the top implanted layer is contacted.

For temperatures above $240 \mathrm{~K}$, the behavior is the one expected for a heavy/light-doped double sheet. The highest sheet resistance a

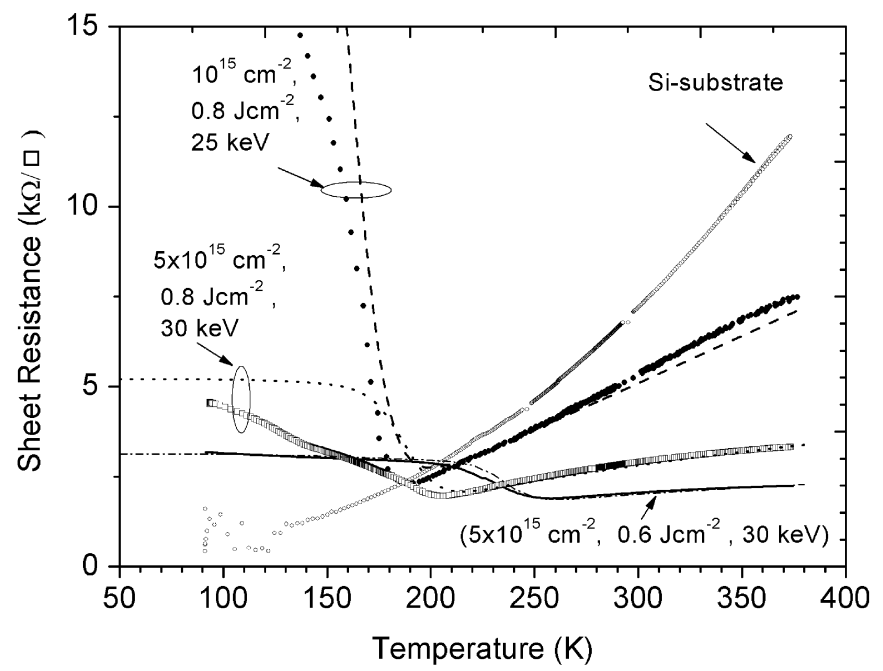

b

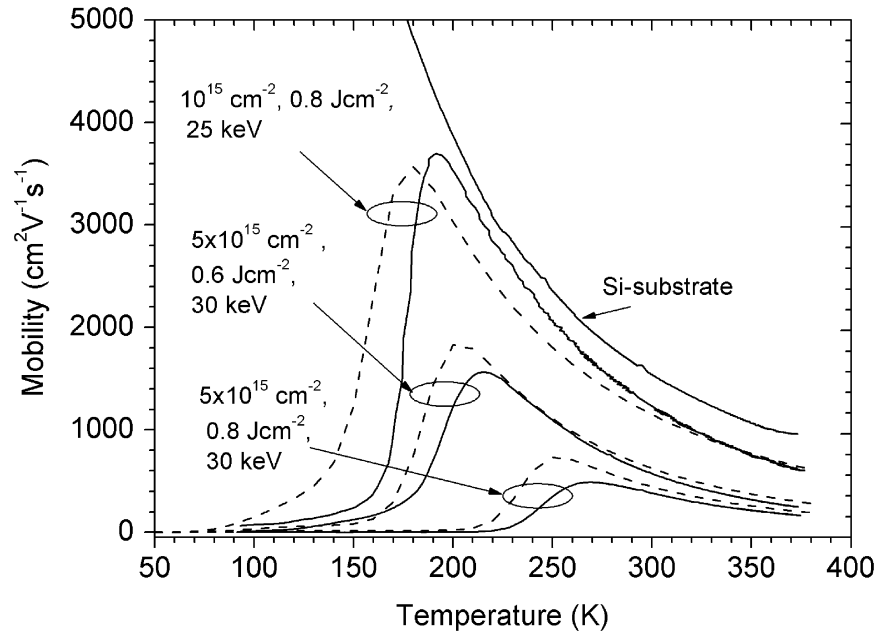

Fig. 2. (a) Sheet resistance and (b) electron Hall mobility vs. the absolute temperature of a silicon wafer with titanium implanted at the surface. The unimplanted substrate data are also presented. Hall mobility is actually negative revealing electron conduction. Dashed lines represent the fit obtained by assuming a factor $F$ that quickly changes from 0 to 1 when the temperature transits through $200 \mathrm{~K}$.

occurs for the unimplanted substrate and increases with the temperature due to the reduction of the mobility, but when there is an implanted layer, it operates in parallel and the sheet resistance is reduced by the conduction in the highly conductive implanted layer. This conduction is the highest for the case of implantation with $5 \times 10^{15} \mathrm{~cm}^{-2} \mathrm{Ti}$ atoms together with the lowest energy laser irradiation $\left(0.6 \mathrm{~J} \mathrm{~cm}^{-1}\right)$ that is deemed to produce less Ti escape.

As for the mobility, of $n$ type, it decreases monotonically in this range because it is dominated by the scattering with phonons whose concentration increases with temperature. Also in the implanted cases the mobility is smaller due to higher scattering with impurities.

At temperatures below $240 \mathrm{~K}$ the behavior of the substrate does not experience any change of tendency, as it is well known to occur, but several odd characteristics appear for the rest of the cases: (a) The sheet resistance of the whole sample is higher than the sheet resistance of the substrate. This is odd because the combined resistance of two branches in parallel cannot exceed the 
resistance of any branch; (b) The mobility has regions in which it increases as the temperature increases. Increasing shapes may be found at temperatures below $90 \mathrm{~K}$ when the scattering by charge particles dominates [31] but this is not likely to occur above these temperatures. In general the mobilities are negative, that is, they are electron-type mobilities, but when the temperature is very low they become very close to zero and then the sign is uncertain. In some cases very small positive mobility seems to have been observed.

The sheet resistance of two layers in parallel, one of them consisting of an ordinary semiconductor with electron and hole conduction and the other being an intermediate band material is given by:

$R_{\|}=\frac{1}{e\left(f_{s} p_{s} \mu_{p s}+f_{b} p_{b} \mu_{p b} F+f_{s} p_{I} \mu_{I}+f_{s} n_{s} \mu_{n s}+f_{b} n_{b} \mu_{n b} F\right) t}$

where the subindices $b$ and $s$ refer to the bulk (substrate) and surface (implanted) sheets, $f_{b} t$ and $f_{s} t$ are the thicknesses of these sheets, the subindex $I$ refers to the carriers in the IB (electrons and holes) which are assumed to be in the surface sheet and $F$ is a factor that equals one when the two sheets are perfectly coupled and zero when the substrate is perfectly decoupled. The nature of this decoupling will be explained later.

Hall voltages appear [32] when the sample is under the influence of a magnetic induction. For the case of having two sheets in parallel and current conduction in the IB in addition to the $V B$ and $C B$, the generalized formula for the Hall mobility (neglecting energy dispersion effects), assuming hole-IB conduction, is given by

$\mu_{H}=\frac{\left(f_{s} \mu_{p s}^{2} p_{s}+f_{b} \mu_{p b}^{2} p_{b} F+f_{s} \mu_{I}^{2} p_{I}-f_{s} \mu_{n s}^{2} n_{s}-f_{b} \mu_{n b}^{2} n_{b} F\right)}{\left(f_{s} p_{s} \mu_{p s}+f_{b} p_{b} \mu_{p b} F+f_{s} p_{I} \mu_{I}+f_{s} n_{s} \mu_{n s}+f_{b} n_{b} \mu_{n b} F\right)}$

In case of electron conduction in the IB, the corresponding term in the numerator should be negative. In Eqs. (1) and (2), several terms may be negligible and in some cases only one term is dominating. For instance, in the substrate, only the terms in $n_{b}$ are important so that $R_{\|}=1 / e n_{b} \mu_{n b} t$ and $\mu_{H}=-\mu_{n b}$.

For explaining the high sheet resistance observed below $240 \mathrm{~K}$ we must admit that the substrate is decoupled, at least to a certain extent ( $F$ small) so that the sheet resistance and possibly the Hall mobility become dominated by the implanted layer. If this happens, the resistance tends to grow at low temperature due to the reduced ionization of the Ti donor level (or band) that is located quite deep in the band gap. This effect does not happen in the substrate because its donor level is very shallow. This band has probably a low mobility as suggested by the large effective mass to be expected from a narrow band. The IB current could be supported by electrons when it is almost fully ionized (at higher temperature) or holes when it is almost full of electrons. In consequence, the sheet resistance is given in this case by $R_{\|}=1 / e\left(f_{s} p_{I} \mu_{I}+f_{b} n_{b} \mu_{n b} F\right) t$. Thus, the sheet resistance may be high.

The measured mobility at low temperature is a combination of the substrate and IB mobilities given by $\mu_{H}=\left(f_{s} \mu_{I}^{2} p_{I}-f_{b} \mu_{n b}^{2} n_{b} F\right) /$ $\left(f_{s} p_{I} \mu_{I}+f_{b} n_{b} \mu_{n b} F\right)$. The fact that the measured mobility is usually negative suggests, even if the IB mobility is positive, that the substrate term, characterized by its high mobility, can dominate even if the conduction is mainly through the IB. But the IB mobility might also be negative. Anyway, it cannot be strictly zero because in this case the measured mobility at very low temperature should have been $\mu_{H}=\mu_{n b}$ which has been measured to be high. This fact is to be stressed because it shows that there is a small but non-zero IB mobility.

\section{Nature of the layer decoupling: formation of an IB- $n$ blocking junction}

In some implanted wafers, we have deposited circular aluminum contacts ( $2 \mathrm{~mm}$ diameter) with guard rings on the front face and a wider contact in the back face in order to investigate the decoupling mechanism. Diode $I V$ curve shapes have appeared at low temperature that, surprisingly, are absent at high temperature. We present the IV curves at low and high temperature in Fig. 3. This diode behavior under reverse bias is the cause of the substrate-layer decoupling.

Although the effect is distributed, the sheet resistance measurements can be explained with Fig. 4. If the diodes are short-circuted as it happens at high temperature, the sheet resistances of the implanted layer and the substrate are measured in parallel but, if they are active, then one of the two diodes is in reverse bias and no current can flow through the substrate.

To explain the nature of the blocking diode in terms of IB formation, we first illustrate in Fig. 5 a schematic of a $n$-IB junction the IB being the relatively deep donor level found. As explained in Ref. [4], the Fermi level in the IB material splits under nonequilibrium conditions into three quasi-Fermi levels, one for the $\mathrm{VB}$ one for the $\mathrm{CB}$ and a third one for the IB.

At high temperature, the electron density equals the donor density, which is totally ionized (or almost) in both sides (this case is not illustrated in Fig. 5). Non-equilibrium situation (as the one

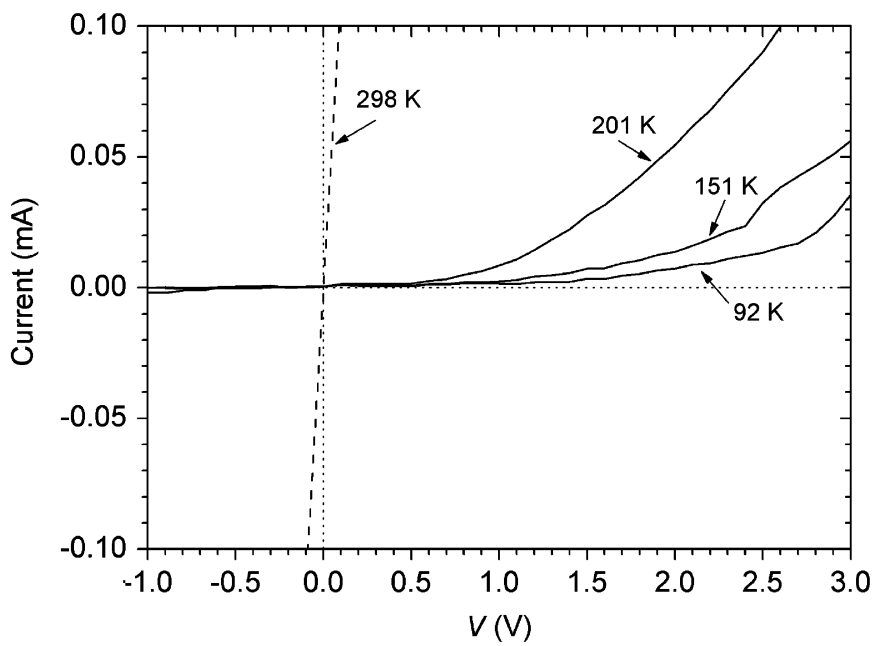

Fig. 3. Current-voltage plots at low and high temperatures obtained when contacting the implanted wafers with front and back contacts and guard rings at the front. At low temperature, the characteristics show the rectifying behavior that is responsible for the decoupling of the implanted layer with respect to the substrate. This behavior disappears at room temperature (298 K).

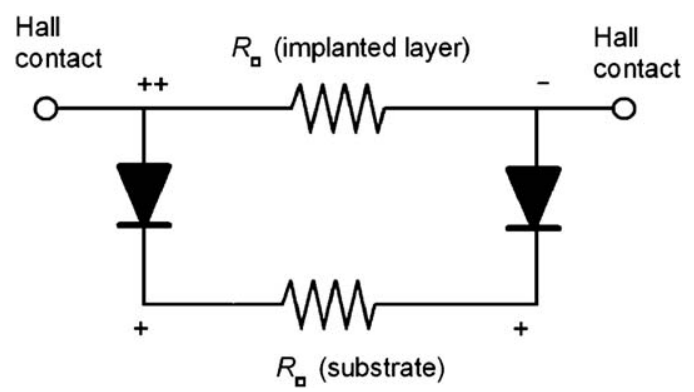

Fig. 4. Schematic of the blocking diode between the substrate and the IB layer in the sheet resistance and Hall effect measurements. At high temperature the diodes are practically short circuits. 
a

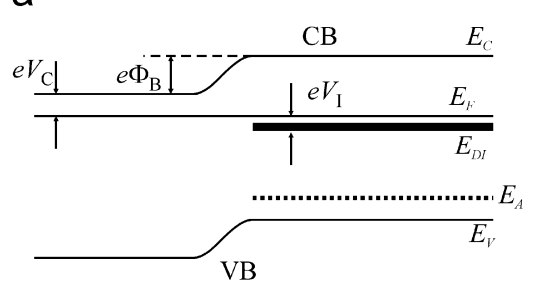

b

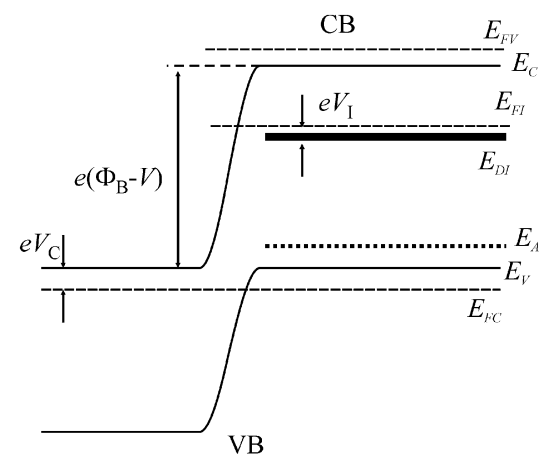

Fig. 5. IB- $n$ junction for compensated semiconductor at low temperature in (a) equilibrium and (b) reverse biased.

introduced during the Hall measurements) does not change this electric-charge equilibrium condition and the barrier between both regions is the one at equilibrium. Assuming no heavy doping effects (that might change the equivalent density of states of the conduction band in substrate and layer, that cancel when they are equal), it is given approximately by $e \phi_{B}=k T \ln \left(N_{D b} / N_{D I}\right)$ where the $N_{D}$ 's are the density of donors in both regions. No voltage can appear in the interface.

The situation changes drastically at low temperature. As before, the quasi-Fermi level for electrons at the substrate side stays pinned to the $\mathrm{CB}$ by $N_{D b}=N_{C} \exp \left(-V_{C} / k T\right) \quad\left(N_{C}\right.$ is the equivalent density of states of the $C B$; see Fig. $5 a$ for the rest of the nomenclature). As for the IB, we assume partial compensation; that is, the existence of a smaller density of states $N_{A I}$ in an acceptor level (fully ionized) that keeps the charge neutrality with the few ionized donors when the electron density has decreased below the acceptor density of states. Nevertheless, it has to be said that this acceptor level is not necessary to explain the changes in the $n$-IB barrier that follows but simplifies the discussion.

According to our assumption, at low temperature the charge neutrality is produced by equating the ionized donor density with the acceptor density. Thus, the IB quasi-Fermi level is pinned to the IB (disregarding for simplicity-level degeneration) by $N_{A I}=N_{D I}$ (exp $\left.\left(V_{I} / k T\right)+1\right)^{-1} \cong N_{D I} \exp \left(-V_{I} / k T\right)$. In equilibrium, with all quasi-Fermi levels together $e \phi_{B}=E_{C}-E_{D I}-V_{C}-V_{I}=E_{C}-E_{D I}-k T \ln \left(N_{D I} N_{C} / N_{A I} N_{D b}\right)$. In non-equilibrium, it is possible to split the quasi-Fermi levels and the barrier will increase together with this splitting so that a voltage $e V=E_{F C}-E_{F I}$ appears in the barrier. Notice that, in reverse bias, that is the case represented in Fig. $5 \mathrm{~b}, V$ is negative ( $E_{F I}$ above $E_{F C}$ ).

By calling $p_{I}=N_{D I}-n_{I}$ the density of ionized donors in the IB, $n_{I}$ being the concentration electrons in the intermediate band, the charge neutrality condition is precisely $N_{A I}=P_{I}$.

Neglecting the generation-recombination processes in the space charge zone, the current across the $n$-IB junction is the sum of the diffusion currents of the minority holes at the substrate side and of the almost minority electrons at the implanted side:

$J \cong \frac{k T \mu_{p}}{L_{p}} \Delta p\left(-x_{n}\right)+\frac{k T \mu_{n}}{L_{n}} \Delta n\left(x_{I}\right)$ where the Einstein relationship for diffusion constants has been used. The L's represent characteristic distances. For long diodes it corresponds to the diffusion length; for short diodes it might be the region thickness if the surface recombination is infinite. The current of the holes in the IB has not to be calculated (in the limit above mentioned) because this current has to be zero since it has no continuity on the base side. $\Delta p\left(-x_{n}\right)$ and $\Delta n\left(x_{I}\right)$ are the hole excess at the $n$-side and electron excess at the implanted region respectively, neighbors to the $n$-IB junction space charge region, and are given by

$\Delta p\left(-x_{n}\right)=\frac{n_{i}^{2}}{N_{D}} \exp \left(\frac{E_{F C}-E_{F V}}{k T}\right)$
$\Delta n\left(-x_{I}\right) \approx \frac{N_{C} N_{D I}}{N_{A I}} \exp \left(\frac{E_{D I}-E_{C}}{k T}\right) \exp \left(\frac{e V}{k T}\right)$

where $n_{i}$ is the silicon intrinsic concentration. Eq. (4) is obtained applying the conventional Shockley's diode model to the $n$-side of the junction. Eq. (5) is obtained by noticing that

$n\left(x_{I}\right) p_{I} \approx N_{C} \exp \left(\frac{E_{F C}-E_{C}}{k T}\right) N_{D I} \exp \left(\frac{E_{D I}-E_{F I}}{k T}\right)$

together with $e V=E_{F C}-E_{F I}$ and $p_{I} \approx N_{A I}$. Substituting $\Delta p\left(-x_{n}\right)$ and $\Delta n\left(x_{I}\right)$ into Eq. (3) leads to

$$
\begin{aligned}
J \cong & \left.\frac{k T \mu_{p}}{L_{p} N_{D}} N_{C} N_{V} \exp \left(-E_{G} / k T\right)\left\{\exp \left[\left(E_{F C}-E_{F V}\right) / k T\right)\right]-1\right\} \\
& +\frac{k T \mu_{n}}{L_{n} N_{A I}} N_{C} N_{D I} \exp \left[-\left(E_{C}-E_{D I}\right) / k T\right][\exp (e V / k T)-1]
\end{aligned}
$$

Note that the decoupling is found when the $n$-IB junction is reverse biased. In this case, $E_{F I}$ is above $E_{F C}$ indicating that the currents are dominated by thermal generation mechanisms. For the same reason $E_{F V}$ should be above $E_{F I}$ as required by the carrier balance in the IB if current in this band is negligible. This would entail that $\exp (e V / k T)$ and $\left.\exp \left[\left(E_{F C}-E_{F V}\right) / k T\right)\right]$ are very small leading to a saturation current. The dominant term of this saturation current is the one with the exponential factor $\exp \left[-\left(E_{C}-E_{D I}\right) / k T\right]$ affected by a low band gap.

An interesting aspect of Eq. (7) when only the second term is retained is that it is the same of a hetero-junction between a $n-S i$ material and a $p$-material of $E_{C}-E_{D}$ band gap, with the same affinity than $\mathrm{Si}$ and a doping $N_{A I}$, provided that only the dominant term, of electron injection into the low band gap material, is retained. The advantage of this similarity is that the Atlas simulation code [33] can be used and the coupled and decoupled situations can be visualized in Fig. 6. At high temperature the equipotential lines show a vertical distribution characteristic of a parallel coupling between substrate and implanted layer. At low temperature they show all the substrate at the same potential proving that the substrate does not carry any current.

\section{Conclusions}

In summary, at room temperature our measurements of sheet resistance and Hall mobility on a heavily $\mathrm{Ti}$ implanted silicon wafer are interpreted rather conventionally on the basis of a deep donor level with a negative Hall mobility in the implanted layer. At low temperatures the substrate is decoupled of this layer by a reverse biased $n$-IB junction and the sheet resistance grows above the one of the substrate while the mobility takes values rather low due in part to the low but not zero mobility of the IB. Because of it, mobility is usually observed as a negative mobility. However, at high temperatures, the IB is almost empty of electrons (almost totally ionized) and the electron mobility observed is the one expected; at low temperature the IB is almost full of electrons and 
a

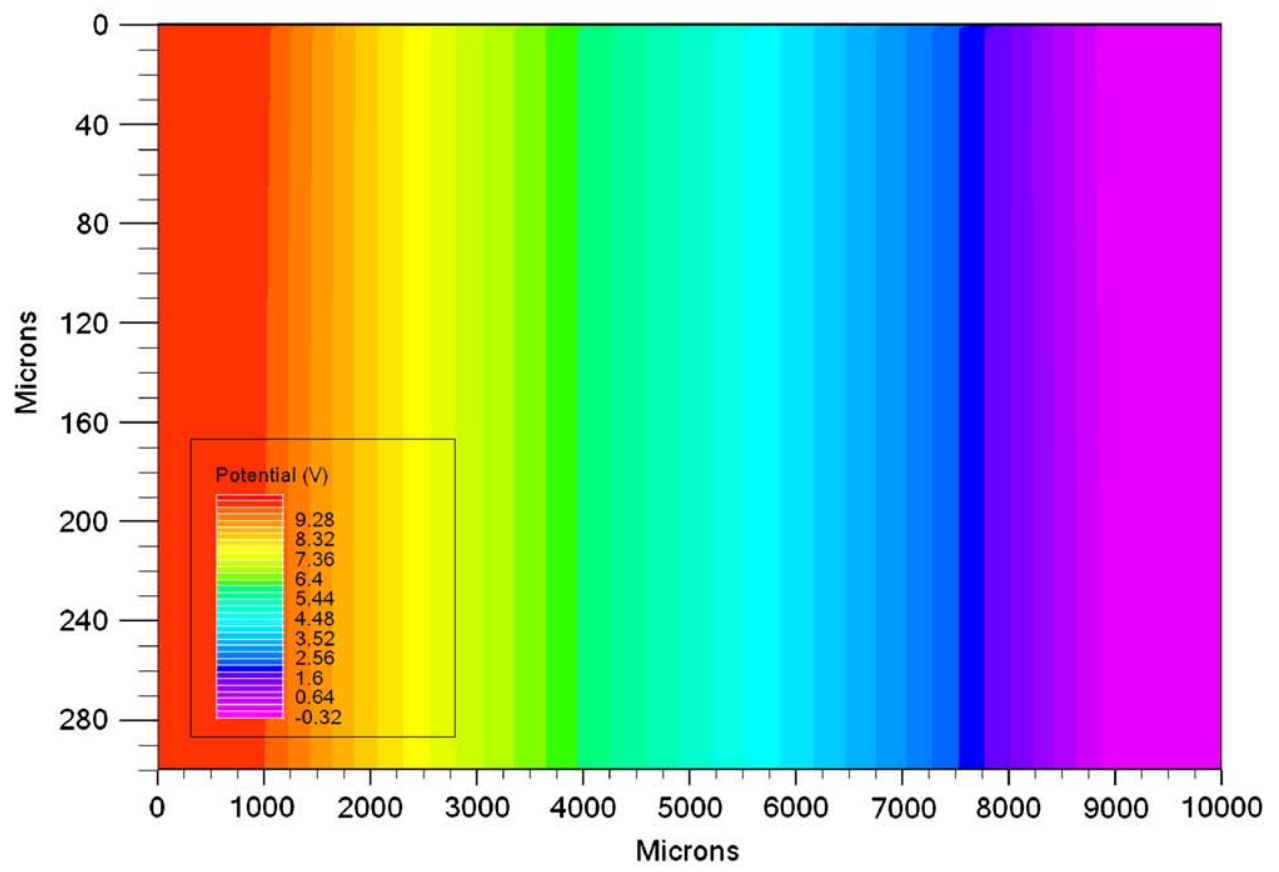

b

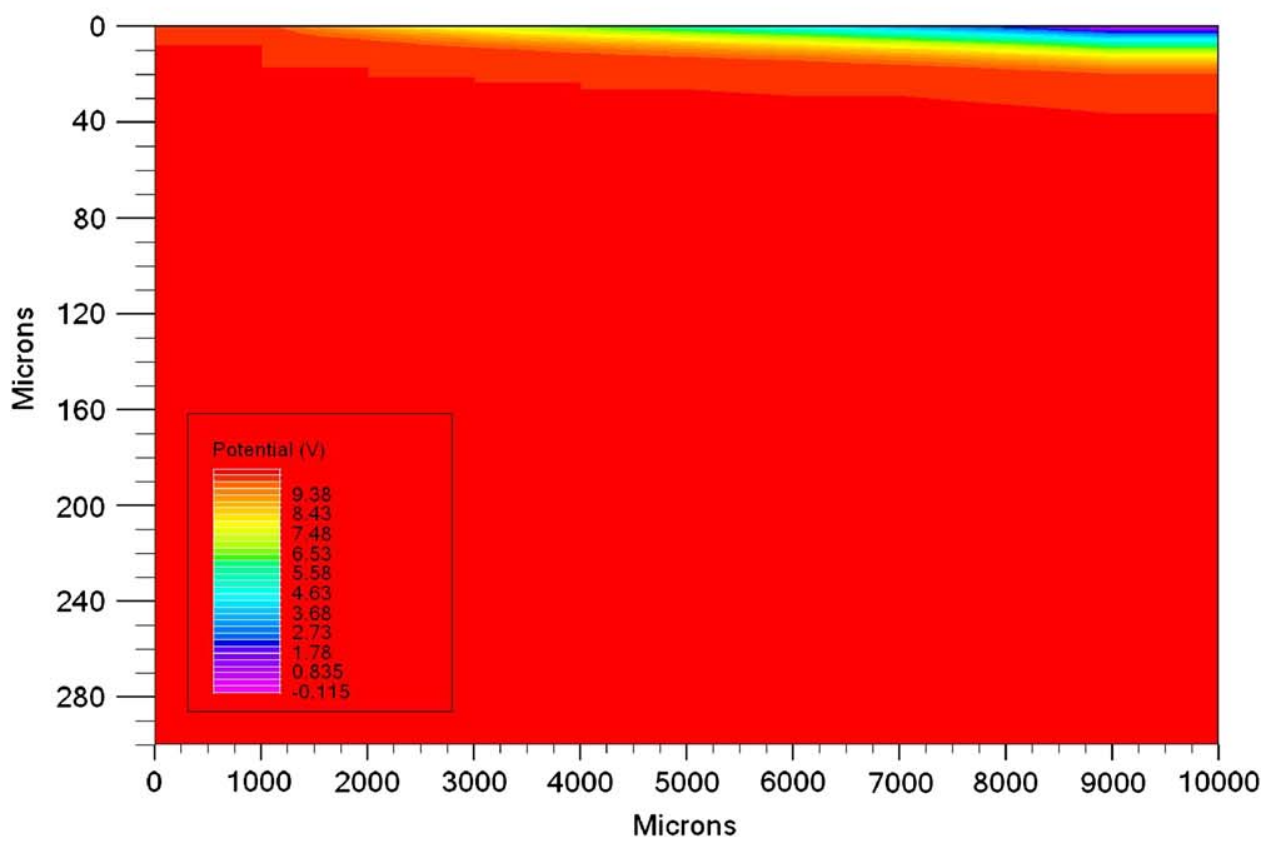

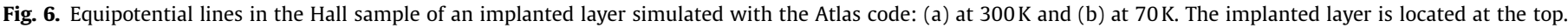

a hole mobility might be expected from theoretical considerations. The existence of an intermediate band with non-zero mobility could also find application for the practical implementation of the selective energy contacts required by hot carrier solar cells $[34,35]$.

\section{Acknowledgments}

This work has been supported by the project IBPOWER, funded by the European Commission under Contract no. 211640, by the Regional Government of Madrid within the project NUMANCIA (S0505/ENE/000310) and by the Spanish National Research Program within the project GENESIS-FV (CSD2006-0004).

\section{References}

[1] W. Shockley, H.J. Queisser, Detailed balance limit of efficiency of $p-n$ junction solar cells, Journal of Applied Physics 32 (1961) 510-519.

[2] G.L. Araujo, A. Marti, Absolute limiting efficiencies for photovoltaic energy conversion, Solar Energy Materials and Solar Cells 33 (1994) 213-240.

[3] A. Luque, A. Martí, Increasing the efficiency of ideal solar cells by photon induced transitions at intermediate levels, Physical Review Letters 78 (1997) 5014-5017.

[4] A. Luque, A. Martí, A metallic intermediate band high efficiency solar cell, Progress in Photovoltaics: Research and Applications. 9 (2001) 73-86.

[5] A. Luque, A. Martí, C. Stanley, N. López, L. Cuadra, D. Zhou, A. Mc-Kee, General equivalent circuit for intermediate band devices: potentials, currents and electroluminescence, Journal of Applied Physics 96 (2004) 903-909.

[6] S.M. Hubbard, C.D. Cress, C.G. Bailey, R.P. Raffaelle, S.G. Bailey, D.M. Wilt, Effect of strain compensation on quantum dot enhanced GaAs solar cells, Applied Physics Letters 92 (2008) 123512-3. 
[7] M.Y. Levy, C. Honsberg, Nanostructured absorbers for multiple transition solar cells, IEEE Transactions on Electron Devices 55 (2008) 706-711.

[8] Q. Shao, A.A. Balandin, A.I. Fedoseyev, M. Turowski, Intermediate-band solar cells based on quantum dot supracrystals, Applied Physics Letters 91 (2007) 163503-3.

[9] G.D. Wei, K.T. Shiu, N.C. Giebink, S.R. Forrest, Thermodynamic limits of quantum photovoltaic cell efficiency, Applied Physics Letters 91 (2007) 223507-223513.

[10] A. Martí, L. Cuadra, A. Luque, Partial filling of a quantum dot intermediate band for solar cells, IEEE Transactions on Electron Devices 48 (2001) 2394-2399.

[11] G. Wei, S.R. Forrest, Intermediate-band solar cells employing quantum dots embedded in an energy fence barrier, Nano Letters 7 (2007) 218-222.

[12] R. Oshima, A. Takata, Y. Okada, Strain-compensated InAs/GaNAs quantum dots for use in high-efficiency solar cells, Applied Physics Letters 93 (2008) 083111-3.

[13] A. Martí, E. Antolin, C.R. Stanley, C.D. Farmer, N. Lopez, P. Diaz, E. Canovas, P.G Linares, A. Luque, Production of photocurrent due to intermediate-toconduction-band transitions: a demonstration of a key operating principle of the intermediate-band solar cell, Physical Review Letters 97 (2006) 247701-247704.

[14] A. Luque, A. Marti, N. Lopez, E. Antolin, E. Canovas, C. Stanley, C. Farmer, L.J Caballero, L. Cuadra, J.L. Balenzategui, Experimental analysis of the quasiFermi level split in quantum dot intermediate-band solar cells, Applied Physics Letters 87 (2005) 083503-083505.

[15] A. Luque, A. Martí, E. Antolín, C. Tablero, Intermediate bands versus levels in non-radiative recombination, Physica B 382 (2006) 320-327.

[16] W. Shockley, W.T. Read, Statistics of the recombination of holes and electrons, Physical Review 87 (1952) 835-842.

[17] R.N. Hall, Electron-hole recombination in germanium, Physical Review 87 (1952) 387.

[18] P. Wahnón, C. Tablero, Ab-initio electronic structure calculations for metallic intermediate band formation in photovoltaic materials, Physical Review B 65 (2002) 1-10.

[19] P. Palacios, J.J. Fernandez, K. Sanchez, J.C. Conesa, P. Wahnon, First-principles investigation of isolated band formation in half-metallic Ti[sub $x] \mathrm{Ga}[\mathrm{sub}$ $1-x] \mathrm{P}(x=0.3125-0.25)$, Physical Review B (Condensed Matter and Materials Physics) 73 (2006) 085206-085208.

[20] P. Palacios, K. Sánchez, J.C. Conesa, J.J. Fernández, P. Wahnón, Theoretical modelling of intermediate band solar cell materials based on metal-doped chalcopyrite compounds, Thin Solid Films 515 (2007) 6280-6284.
[21] A. Marti, D. Fuertes-Marron, A. Luque, Evaluation of the efficiency potential of intermediate band solar cells based on thin-film chalcopyrite materials, Journal of Applied Physics 103 (2008) 073706-6.

[22] A. Rohatgi, J.R. Davis, R.H. Hopkins, P. Raichoudhury, P.G. McMullin, J.R. McCormick, Effect of titanium, copper and iron on silicon solar-cells, SolidState Electronics 23 (1980) 415-419.

[23] J.W. Chen, A.G. Milnes, A. Rohatgi, Titanium in silicon as a deep level impurity, Solid-State Electronics 22 (1979) 801-808.

[24] A.C. Wang, C.T. Sah, Complete Electrical characterization of recombination properties of titanium in silicon, Journal of Applied Physics 56 (1984) $1021-1031$

[25] D. Mathiot, S. Hocine, Titanium-related deep levels in silicon: a reexamination, Journal of Applied Physics 66 (1989) 5862-5867.

[26] M. Okuyama, N. Matsunaga, J.-W. Chen, A.G. Milnes, Photoionization crosssections and energy levels of gold, iron, platinum, silver, and titanium in silicon, Journal of Electronic Materials 8 (1979) 501-515.

[27] L. Tilly, H.G. Grimmeiss, H. Pettersson, K. Schmalz, K. Tittelbach, H. Kerkow, Electrical and optical properties of titanium-related centers in silicon, Physical Review B 43 (1991) 9171-9177.

[28] J. Olea, M. Toledano-Luque, D. Pastor, G. Gonzalez-Diaz, I. Martil, Titanium doped silicon layers with very high concentration, Journal of Applied Physics 104 (2008) 016103-016105.

[29] C.W. White, S.R. Wilson, B.R. Appleton, F.W. Young Jr., Supersaturated substitutional alloys formed by ion implantation and pulsed laser annealing of group-III and group-V dopants in silicon, Journal of Applied Physics 51 (1980) 738-749.

[30] K.M. Yu, W. Walukiewicz, J. Wu, W. Shan, J.W. Beeman, M.A. Scarpulla, O.D. Dubon, P. Becla, Diluted II-VI oxide semiconductors with multiple band gaps, Physical Review Letters 91 (2003) 246403-246404.

[31] K.W. Boer, Survey of Semiconductor Physics, Van Nostrand Reinhold, New York, 1990, pp. 792-797.

[32] R.L. Petritz, Theory of an experiment for measuring the mobility and density of carriers in the space-charge region of a semiconductor surface, Physical Review 110 (1958) 1254-1262.

[33] ATLAS, Device simulator framework distributed by Silvaco Data Systems Inc., 4701 Patrick Henry Drive, Bldg\#6, Santa Clara, CA.

[34] G.J. Conibeer, C.W. Jiang, D. König, S. Shrestha, T. Walsh, M.A. Green, Selective energy contacts for hot carrier solar cells, Thin Solid Films 516 (2008) 6968-6973.

[35] P. Wurfel, Solar energy conversion with hot electrons from impact ionisation, Solar Energy Materials and Solar Cells 46 (1997) 43-52. 